\title{
Assessment of Neuroglial Relationships under Photodynamic Treatment Using Fluorescent Visualization of Giant Axons in Crayfish Ventral Nerve Cord
}

\author{
Evgeny Duz and Mikhail S Kolosov* \\ Southern federal university, Rosto von Don, Russia \\ Received: May 28, 2018; Published: June 05, 2018 \\ *Corresponding author: Mikhail S Kolosov, Southern federal University, Rostov-on-Don, Russia
}

\begin{abstract}
There are lots of publications last time about important role of neuroglial interactions in normal physiology of nervous system. Some of them infer that neuroglial interactions may support survival of neuronal and glial cells. Study the mechanisms of neuroglial interactions needs convenient models which are simple nervous system. They could be easily isolated and studied while retain natural neuroglial relations. The purpose of present work was to estimate glia protective properties of giant axons in simple model - crayfish ventral nerve cord, upon photo oxidative stress. To better visualize the axons shape and reveal the periaxonal glial cells, we were filling of the axons with fluorescent label by means of specially devised microinjector. It enabled us to reveal the glial cells which are in close proximity to the axons. Such glial cells were found to have increased survival in compare to more distant ones from the axons under oxidative stress. The obtained data indicate that the crayfish giant axons have glia protective properties, which molecular mechanisms have to be studied.
\end{abstract}

Keywords: Neuroglial Interaction; Oxidative Stress; Glia Protection; Piezo-Driven Microinjection; Neuronal Tracing

\section{Introduction}

Study of neuroglial interactions under nerve tissue injury needs adequate models and instruments. Neuroglial interactions may affect the survival of neurons and glial cells $[1,2]$. So, cell survival rate could be used as an indicator of cytoprotective interactions between neuronal and glial cells. To deepen our understanding of nuroglial interactions it is desirable together with estimation of live/dead status of the cells to have ability to assess neuroglial morphological relations in the sample, because structural intercellular relations provide the basis for the functional ones. Both this tasks seemed us to be done simultaneously with fluorescent microscopy, combining estimation of live/dead status of neuronal and glial cells [3] with cell morphology visualization by direct intracy to plasmic delivery of fluorescent markers using microinjection [4,5]. Thus, the purpose of our largely methodical work was, using nerve tissue preparation convenient for intraneuronal microinjection, to solve two problems:

a) To define axons morphology, and reveal periaxonal glial cells

b) To estimate protective properties of axons for glial cells, observing the survival of periaxonal glial cells during photody namically generated oxidative stress. In other words, the goal of our study was to examine whether tight morphological neuroglial relations promote survival of periaxonal glial cells during oxidative damage of nerve tissue.

\section{Materials and Methods}

As a model object to study influence of neuroglial structural relations on survival of glial cells we used isolated ventral nerve cord (VNC) from freshwater crayfish Astacus. Neighboring ganglia in the ventral nerve cord connected by long connectives (up to $2 \mathrm{~mm}$ ) which include axons of nerve cells enwrapping with multilayer glial sheaths. Among the axons there are two pairs of giant ones go along each connective [6]. Relatively large diameter of giant axons (50 $\mu \mathrm{m}$ mean) made this object well-suitable for microscopy examination. Oxidative stress was generated photodynamically by 30 minirradianion of VNC with laser $\left(670 \mathrm{~nm} ; 0,1 \mathrm{~W} / \mathrm{cm}^{2}\right)$ after 1 hour incubation with $1 \mathrm{uM}$ alumophtalocyanine Photosens.

Survival rate of glial cells surrounding the axons in connectives was estimated 5 hours after irradiation by fluorescence microscopy of VNC, which were stained with propidium iodide (PI) and Hoechst 33342 (Hoechst) for visualization of necrotic and living cells [3], re- 
spectively. While this fluorochromes could be excited simultaneously by UV light (340-380 nm), they have emission maxima at opposite parts of visible spectra - red and blue, respectively. That makes cell nuclei stained by (PI) and Hoechst easily distinguished. For the giant axons morphology visualization we inject them with solution of quantum dots Qdot 655 (Invitrogen, USA) by specially designed microinjector. As a base of the injector we used Chambers-Kopac construction [7] with mechanical feed of micro capillary. However, since giant axons in VNC surrounded by multilayer glial sheath, for its easier piercing Chambers-Kopac construction was combining with tube shaped piezoelectric transducer, which transmit micro vibration at the injection micro capillary. The frequency provided by pulse generator was $25 \mathrm{kHz}$. Such ultrasound vibration of the capillary in work on oocytes [8] reduced deformation of plasma membrane and zona pellucida preceding cell puncture.

It was of importance for our case, since piercing of the axons only by manual feeding of microcapillary (without of micro vibrations) produced considerable glial sheaths deformations prior to puncture, and increase risk of through perforation of the axons (microcapillary fed to them manually by approximately 45 degrees angle, (Figure 1). To lower a risk of the microcapillary break during the piercing of mulylayer glial envelope we used glass micro capillary with relatively thick tips (5-10 um diameter). In some cases the tips of the capillary were beveled at $1 \mu \mathrm{m}$ grade diamond lapping film 662XW (3M, USA) attached to the surface of rotating computer hard drive. Hydraulic system - syringe which was fed by micrometer and connected with the injector by flexible plastic tube with Vaseline oil, was used only to avoid clogging of the capillary - the solution of quantum dots was in capillary at small positive pressure. The injection itself was provided just by capillary microvibration. Injection was followed by short fixation with glutaraldehyde for preserving structure of preparation. Microscopy was carried out just in experimental chamber using Nikon FN1 fluorescent microscope with 330-380 nm excitation and $420 \mathrm{~nm}$ long pass barrier filter cube.

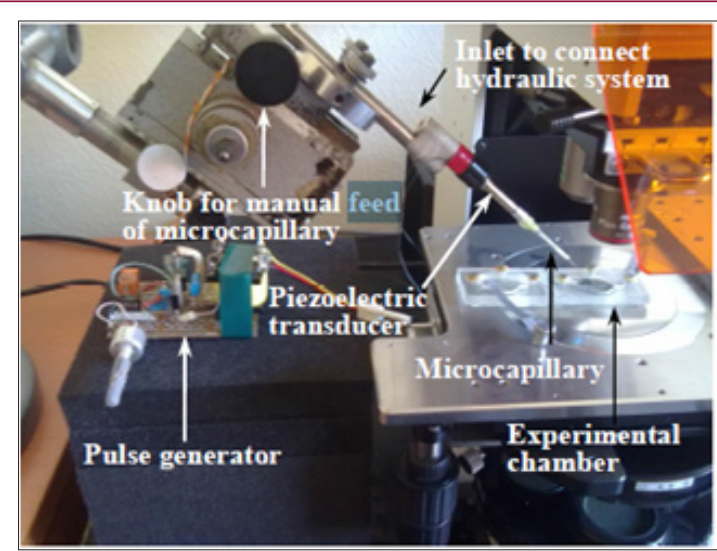

Figure 1: Piezoelectric-enabled microinjector which we devised for visualization of the giant axons.

Filling of giant axons with quantum dots Qdot 655 solution made a background at which one should to distinguish live and necrotic cells, stained with Hoechst and PI, respectively. To do this correctly it need spectral detection of fluorescent image or, because in our case PI and Qdot 655 have similar spectral maximums - some other way to quantitavely estimate the level of PI in the nuclei of cells overlaid the axon background. Our approach was as follow. It is known that nuclear stains PI и Hoechst - interact with DNA in different ways: PI -intercallator [9], but Hoechst binds to minor groove of DNA [10]. Despite the stains has different sites of binding to DNA we had noticed that these bindings are competitive. This was revealed in procedure of sequential staining (PI, than Hoechst, as we used in our protocol), when nuclei of necrotic cells with impaired plasma membrane, pre-stained with PI, bound Hoechst in less amounts in compare withliving cells, which were not pre-stained

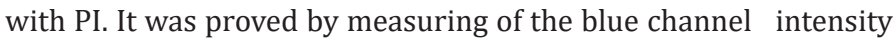
of initial RGB images of cell nuclei (Figure 2). Average intensity of blue-channel pixels for living cells was higher than one for the dead cells (Figure 2D). This observation enabled us to judge about presence of PI in glial nuclei, which overlaid Qdot 655-filled axons, just by comparison of their blue channel intensity with respective values for live (Hoechst-binding) and necrotic (PI+Hoechst-binding) cells in the preparation.

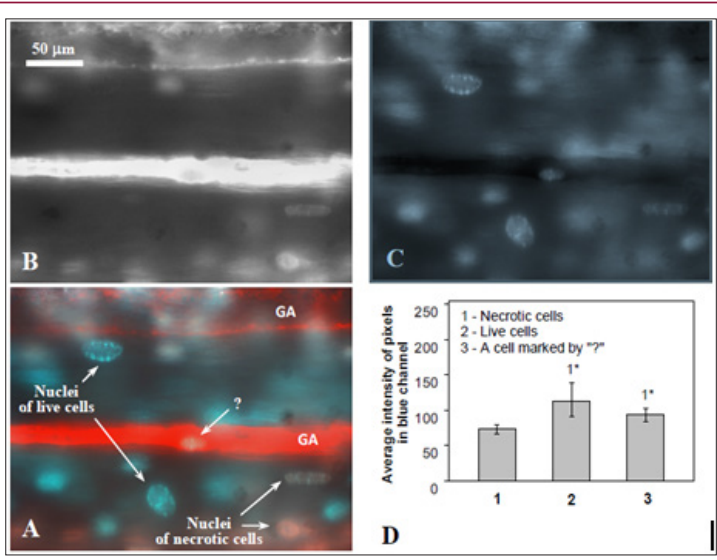

Figure 2: On the approach to define propidium iodide in cell nuclei overlaying the axons stained with Qdot655. A - giant axons (GA) with surrounding glial cells in crayfish ventral nerve cord (fluorescence microscopy). Red - giant axons filled with Qdot 655, blue - nuclei of live glial cells stained with Hoechst, pink - nuclei of necrotic glia stained with PI + Hoechst. By "?" pointed a glial cell overlaid the axon which staining (blue or pink) is not easy to define by the image. B, C - red and blue channels of image A, respectively, in RGB color model. D - average intensity of blue cannel pixels of image of different glial cells: 1necrotic, 2- live, and 3 - one cell pointed at image A with "?". The latter glial cell was recognized as alive since average level of its blue cannel pixels was higher then in necrotic cells, but did not statistically different form the level of living glial cells. * $-\mathrm{p}<0,05$.

\section{Results}

Our piezoelectric-enabled microinjector facilitate piercing of glial sheaths enwrapping giant axons, and promote filling of the axons with fluorescent label without risk of axon damage as a result of overfilling - such thing frequently happen during the injection by pressure. From the site of piercing fluorescent label diffuse in both 
directions of giant axons, revealing their linear morphology along whole nerve connective (Figure 3A). Quantum dots diffusing along giant axons had reached ganglia, where they revealed more complex structures (Figure 3B). It is interestingly that the dye passed through the ganglia, and visualized at the exit the same linear structures as corresponded to the giant axons at the entrance to ganglia. Fluorescence microscopy after the microinjection visualized shape and borders of the axons better than bright field microscopy (Figure 4). Using this approach we found glial cells with nuclei surrounded by halos, which were obviously formed by ununiformity of the axon surface (Figure 4B). This observation enabled us to consider such glial cells as immersed in the axon surface and having direct contacts with axons. By this data we inferred that such cells being in tight structural relations with axons could also have eminent functional neuroglial interrelation, for example which affected on glial cell survival.

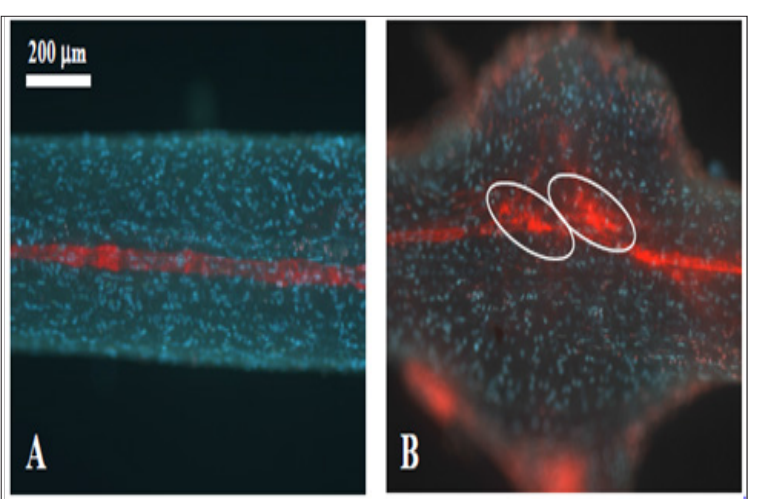

Figure 3: Morphology of the medial giant axon and its projection in fifth abdominal ganglion (fluorescence microscopy). A - crayfish ventral nerve cord connective. B fifth abdominal ganglion (ellipses point two neighbouring non-linear structure which were revealed when fluorescent label, injected in medial giant axon, was passing through the ganglion). Red - Qdot 655 injected in GA. Blue - nuclei of live glial cells stained with Hoechst. Pink - nuclei of necrotic glia stained with PI + Hoechst.

It was interesting to compare post-oxidative stress survival of glial cells in VNC at different remoteness from the giant axons. To do this we distinguished percent of necrotic glial cells at the 3 different locations on images of preparations: at the axons' surface ("immerced cells"), and in two zones along axons - within two diameters of axon (periaxonal zone) and outside periaxonal zone till the border of captured image (distant zone , Figure 4B \& 4C). In control, untreated group of preparations we did not observe necrotic glial cells neither among "immerced cells", nor in the periaxonal zone (Figure 5). Necrosis in the distant zone was also very small (about $5 \%$ ). Photo oxidative stress increased necrosis of glial cells in the periaxonal zone in 5 hours after the treatment up to $30 \%$. Glial necrosis in distant zone was raised even more - up to $80 \%$. But it was interesting that we had not detected a one necrotic glial cell among "immerced cells" in axon surface. Such character of distribution of necrotic glial cells around giant axons after the injury suggests about presence in crayfish ventral nerve cord of neuroglial interactions, which protect glial cells from photo oxidative damage.

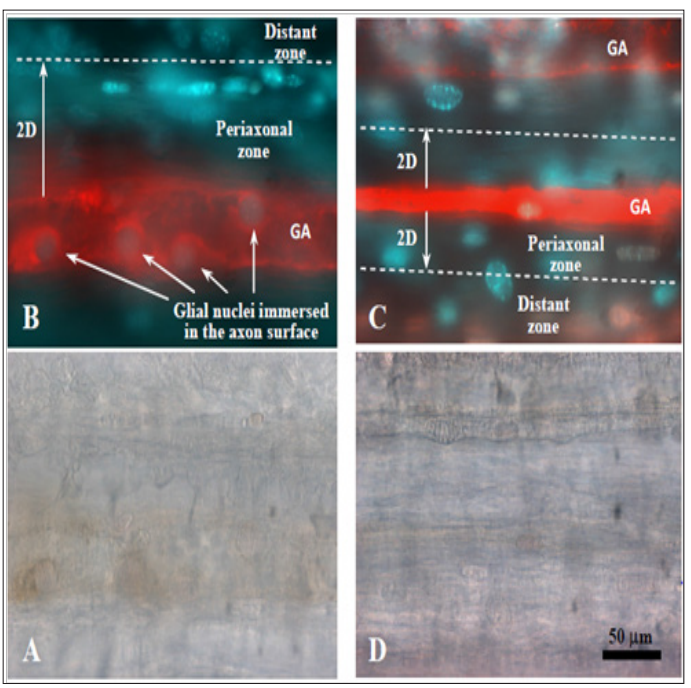

Figure 4: Two modes of visualization of the gaint axons and periaxonal glial cells in crayfish ventral nerve cord. I (A, D) - transmitted light microscopy, II (B, C) fluorescence microscopy (Red - Qdot 655 injected in GA. Blue - nuclei of live glial cells stained with Hoechst. Pink - nuclei of necrotic glia with PI + Hoechst). A, B - control preparation without treatment. C, D - preparation 5 hours after photodynamically generated oxidative stress. Dotted line limits virtual periaxonal zone around giant axons (GA) by two axon diameters (2D).

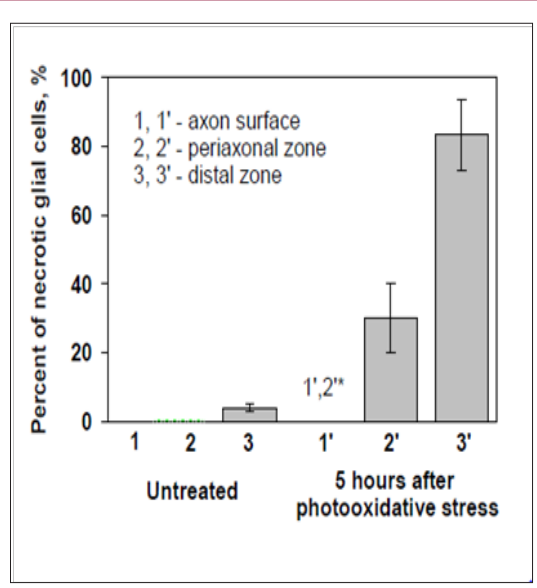

Figure 5: Necrosis of the glial cells at different remoteness from the giant axons ( 5 hours after photooxidative stress). * $-\mathrm{p}<0,05$.

\section{Conclusion}

Microinjection technique, which we are used, successfully visualized morphology of giant axons in crayfish nerve cord. Alternative techniques of cell shape visualization within tissues like labeling of cell plasma membranes with lipophilic fluorescent tracers are time-consuming and could take from several days to weeks [11]. Reconstruction of cell morphology using fluorescence of fixative agent - formalin, which tends to accumulate more in intercellular space than in cells, is complicated and needs confocal or ultra microscopy [12]. Our approach of selective axons morphology visualization by microinjection takes just several minutes. The monitoring 
of the process and final imaging were done with conventional wide field fluorescent microscope.

Using of micro vibrations of the micro capillary for the injection procedure enable rapid diffusion of fluorescent marker along axons and did not need application of extra positive intra capillary pressure, which is not easy to control. First systemic studies of giant axons in the ventral nerve cord of decapods crustacean related to 1920th and name of George Jonson [6]. However, despite long history of study of different aspects of ventral nerve cord anatomy, the questions about location of related to giant axons cell stomata and whether each giant axon incessantly run along whole VNC and belongs to only one neuron (or belong to different connected neurons) are still open. In our study fluorescent marker injected into giant axon prior to ganglion passed it through, revealing at the exit linear shape of axon similar to the axon shape at the entrance to the ganglion. However, just inside the ganglion the marker revealed not a linear pattern, but two neighboring groups of non-linear structures. They could be of axon terminals and dendritic tree of separate neurons which have contacts in ganglion. To obtain more detailed data on morphological architecture of these contacts, as well as for to clarify morphological relations of neuron cell bodies and glial cells in ganglia it will be useful to do confocal or multiphoton examination of the crayfish ventral nerve cord.

Observed phenomenon of increased survival of the glial cells in the direct proximity from neurons' axons may have several suppositional reasons. In our previous work with slightly different design of experiment [13], where we had seen the same effect in the crayfish ventral nerve cord under photo oxidation, we excluded such possible reasons of the phenomena as lack of photosensitizer and light power in the deep nerve tissue layers near the axons. Therefore the observed effect of increased survival of the glial cells as one approached to the giant axons, could be consequence of glia protective neuroglial interactions in the crayfish VNC. Obtained results seem us of special importance because existing literature on support of survival between neural and glial cells relate mainly to embryonic and neonatal periods of individual development, when nerve tissue are under formation [2,14]. Before, we have already report on neuron-dependent survival of glial cells in stretch receptor of adult crayfish [15]. The obtained in present study data once again indicate to existence of support of survival between neural and glial cells in adult nerve tissue, particularly in freshwater crayfish astacus. The molecular mechanisms of the phenomenon are still to be elucidated.

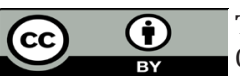

This work is licensed under Creative Commons Attribution 4.0 License

Submission Link: https://biomedres.us/submit-manuscript.php

\section{References}

1. Largo C, Cuevas P, Herreras O (1996) "Is glia disfunction the initial cause of neuronal death in ischemic penumbra?". Neurol Res 18(5): 445-448.

2. Kopp DM, Trachtenberg JT, Thompson WJ (1997) “Glial growth factor rescues Schwann cells of mechanoreceptors from denervation-induced apoptosis." J Neurosci 17(17): 6697-6706.

3. Kolosov MS, Bragin DE, Kohany A, Uzdensky AB (2003) "Photodynamic injury of isolated neuron and satellite glial cells: Morphological study." IEEE Journal of Selected Topics in Quantum Electronics 9(2): 337-342.

4. Meller K, Krah K, Theiss C (2005) "Dye coupling in Purkinje cells of organotypic slice cultures.” Brain Res Dev Brain Res 160(1): 101-105.

5. Goldstein RS, Weiss KR, Schwartz JH (1982) "Intraneuronal injection of horseradish peroxidase labels glial cells associated with the axons of the giant metacerebral neuron of Aplysia". J Neurosci 2(11): 1567-1577.

6. Johnson G (1924) "Giant nerve fibers in crustaceans with special reference to cambarus and palaemonetes". The Journal of Comparative Neurology 36(4): 323-373.

7. Chambers R, Kopac M (1950) “Micrurgical technique for the study of cellular phenomena" in [McClung's Handbook of Microscopical Technique]. Hoeber, New York pp: 492-543.

8. Huang HB, Su H, Chen HY, Mills JK (2011) “Piezoelectric Driven Non-toxic Injector for Automated Cell Manipulation." Studies in Health Technology and Informatics 163: Medicine Meets Virtual Reality 18. IOS Press pp: 231-235.

9. Wilson WD, Wang YH, Krishnamoorthy CR, Smith JC (1986) “Intercalators as probes of DNA conformation: propidium binding to alternating and non-alternating polymers containing guanine." Chem Biol Interact 58(1): 41-56.

10. Chen, AY, Yu C, Gatto B, Liu LF (1993) "DNA minor groove-binding ligands: A different class of mammalian DNA topoisomerase I inhibitors." Proc Natl Acad Sci USA: Pharmacology 90: 8131-8135.

11. Swift MJ, Crago PE, Grill WM (2005) "Applied electric fields accelerate the diffusion rate and increase the diffusion distance of DiI in fixed tissue." J Neurosci Methods 141: 155-163.

12. Leischner U, Schierloh A, Zieglgänsberger W, Dodt HU (2010) “FormalinInduced Fluorescence Reveals Cell Shape and Morphology in Biological Tissue Samples." PLoS ONE 5(4): e10391.

13. Kolosov MS, Duz E, Uzdensky AB (2011) "Photodynamic damage of glial cells in crayfish ventral nerve cord" Proceedings of SPIE 7999. 79990N-79990N.

14. Syroid DE, Maycox PR, Burrola PG, Liu N, Wen D, et al. (1996) “Cell death in the Schwann cell lineage and its regulation by neuregulin." Proc Natl Acad Sci U S A 93(17): 9229-9234.

15. Kolosov M, Uzdensky A (2006) “Crayfish mechanoreceptor neuron prevents photoinduced apoptosis of satellite glial cells." Brain Research Bulletin 69(5): 495-500.

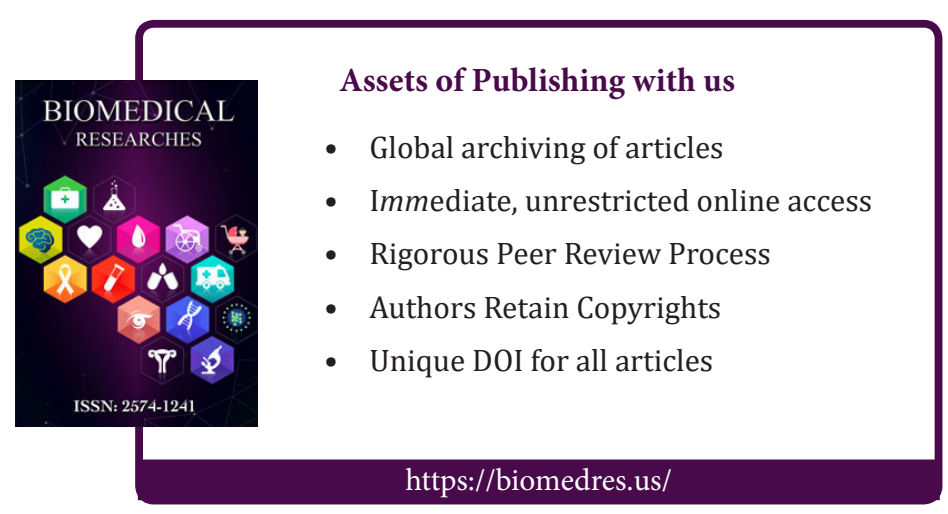

